\title{
Gaussian Beam Diffraction and Self-Focusing in Weakly Anisotropic and Dissipative Nonlinear Plasma
}

\author{
P. BERCZYNSKI ${ }^{a}$, S. BERCZYNSKI ${ }^{b}$ AND YU.A. KRAVTSOV ${ }^{c, d}$ \\ ${ }^{a}$ Institute of Physics, ${ }^{b}$ Department of Mechanical Engineering and Mechatronics \\ West Pomeranian University of Technology, al. Piastów 19, 70-310 Szczecin, Poland \\ ${ }^{c}$ Institute of Physics, Maritime University of Szczecin, 70-500 Szczecin, Poland \\ ${ }^{d}$ Space Research Institute, Russian Academy of Sciences, Moscow 117 997, Russia
}

(Received May 20, 2013; revised version November 20, 2013; in final form December 14, 2013)

The paper presents a simple and effective method to calculate polarization and diffraction of the Gaussian beam in nonlinear and weakly dissipative plasma. The presented approach is a combination of quasi-isotropic approximation of geometric optics with complex geometrical optics. Quasi-isotropic approximation describes the evolution of polarization vector reducing the Maxwell equations to coupled ordinary differential equations of the first order for the transverse components of the electromagnetic field. Complex geometrical optics describes the Gaussian beam diffraction and self-focusing and deals with ordinary differential equations for Gaussian beam width, wave front curvature, and amplitude evolution. As a result, the quasi-isotropic approximation + complex geometrical optics combination reduces the problem of diffraction and polarization evolution of an electromagnetic beam to the solution of the ordinary differential equations, which enable to prepare fast and effective numerical algorithms. Using combined complex geometrical optics/quasi-isotropic approximation for weakly anisotropic plasma, we assume that nonlinearity of anisotropy tensor is small and we restrict ourselves to considering only isotropic nonlinearity. The quasi-isotropic approximation effectively describes the evolution of microwave and IR electromagnetic beams in polarimetric and interferometric measurements in thermonuclear reactors and the complex geometrical optics method can be applied for modeling of electron cyclotron absorption and current drive in tokamaks.

DOI: 10.12693/APhysPolA.125.39

PACS 52.35.- g, 52.35.Mw, 52.38. Hb

\section{Introduction}

Electromagnetic wave propagation in weakly anisotropic plasma is described by quasi-isotropic approximation (QIA) of the geometrical optics method [1-4]. This method reduces the Maxwell equations to the coupled differential equations of the first order. As a modification of geometrical optics, the QIA approach does not describe diffraction processes, which might distort the el-m waves propagating in the nonlinear magnetized plasma. However, there exists an opportunity to describe diffraction processes by the way of combining the QIA equations with the equations of complex geometrical optics (CGO) which adequately describe diffraction processes for Gaussian beams.

The QIA method possesses three equivalent forms: complex polarization angle (CPA) [5, 6], complex amplitude ratio (CAR) [7], and angular variable technique (AVT) $[8,9]$. The method is applied to the problem of electromagnetic wave propagation in the magnetized cylindrical plasma.

The CGO has two equivalent forms: the ray-based form, which deals with complex rays $[10-16]$, that is with trajectories in a complex space, and the eikonal-based form, which uses complex eikonal instead of complex rays [16]. An important feature of $\mathrm{CGO}$ is its ability to describe Gaussian beam (GB) diffraction in both ray-based and eikonal-based approaches. This paper describes the advantages of the eikonal-based form of CGO for description of Gaussian beam diffraction and self-focusing in nonlinear lossy plasma. Recently, eikonal-based CGO method has been generalized for the case of inhomogeneous [17-19] and nonlinear Kerr type media [20]. In the range of sub-millimeter microwaves and moderate infrared waves tokamak plasma (including JET, ITER) is weakly anisotropic, weakly inhomogeneous and weakly nonlinear. To authors knowledge there is not alternative approach to that presented in this paper.

\section{Basic equations of QIA}

Let us present the dielectric tensor $\varepsilon_{i j}$ as a sum of isotropic and anisotropic parts

$$
\varepsilon_{i j}(\boldsymbol{r})=\varepsilon_{0}(\boldsymbol{r}) \delta_{i j}+v_{i j}(\boldsymbol{r}),
$$

where $\delta_{i j}$ is a unit tensor. For a weakly anisotropic medium, tensor $\nu_{i j}$ is small as compared with "isotropic" permittivity $\varepsilon_{0}$. We involve an "anisotropic" small parameter

$$
\mu_{\mathrm{A}}=\frac{\max \left|\nu_{i j}\right|}{\varepsilon_{0}} \ll 1,
$$

which is additional to the conventional "geometrical" small parameter

$$
\mu_{\mathrm{GO}}=\frac{\lambda_{0}}{L} \ll 1 \text {. }
$$

Here $\lambda_{0}$ is the beam wavelength and $L \sim 1 /\left|\nabla \varepsilon_{0}\right|$ is a characteristic scale of the medium inhomogeneity.

The QIA equations can be arrived at by expanding solution of the Maxwell equations in powers of the combined small parameter

$$
\mu=\max \left(\mu_{\mathrm{A}}, \mu_{\mathrm{GO}}\right) \text {. }
$$

In the lowest order in this parameter, QIA leads to the 
wave field in the form [1-4]:

$$
\boldsymbol{E}=\boldsymbol{\Gamma} A \exp \left(\mathrm{i} k_{0} \psi\right),
$$

where $A \exp \left(\mathrm{i} k_{0} \psi\right)$ is a scalar wave field in an isotropic medium of permittivity $\varepsilon_{0}, \boldsymbol{\Gamma}$ is a polarization vector and $k_{0}=2 \pi / \lambda_{0}$ is the wave number. The amplitude $A(\boldsymbol{r})$ and eikonal $\psi(\boldsymbol{r})$ are complex-valued and satisfy the equations of CGO, which are presented in Sect. 3. The polarization vector $\boldsymbol{\Gamma}$ is orthogonal to the unit vector $\boldsymbol{l}$, tangent to the ray, and can be presented as superposition

$$
\Gamma=\Gamma_{1} e_{1}+\Gamma_{2} e_{2}, \quad e_{1} \perp e_{2} \perp l,
$$

where $e_{1}$ and $e_{2}$ are the unit vectors orthogonal to the ray.

Convenient choice of the unit vectors $e_{1,2}$ belongs to Popov [21], who introduced the orthogonal curvilinear coordinates system, which performs parallel transport of the vector wave field $\boldsymbol{E}$ along the ray (so-named Popov's torsionless coordinate system). According to [21], the unit vectors $e_{\mathbf{1}, \mathbf{2}}$ satisfy the equations

$$
\frac{\mathrm{d} \boldsymbol{e}_{i}}{\mathrm{~d} \tau}=-\frac{1}{2}\left(\boldsymbol{e}_{i} \cdot \nabla \ln \varepsilon_{0}\right) \boldsymbol{l}, \quad i=1,2,
$$

where $\tau$ is a parameter changing along the ray, connected with the arc length $\sigma$ by the relation $\mathrm{d} \tau=\mathrm{d} \sigma / \sqrt{\varepsilon_{0}}$. Within the framework of the Popov coordinate system, the QIA equations take the following form:

$$
\begin{aligned}
\frac{\mathrm{d} \Gamma_{1}}{\mathrm{~d} \tau} & =\frac{\mathrm{i}}{2} k_{0}\left(\nu_{11} \Gamma_{1}+\nu_{12} \Gamma_{2}\right), \\
\frac{\mathrm{d} \Gamma_{2}}{\mathrm{~d} \tau} & =\frac{\mathrm{i}}{2} k_{0}\left(\nu_{21} \Gamma_{1}+\nu_{22} \Gamma_{2}\right) .
\end{aligned}
$$

Thus, within the framework of QIA approach, the description of polarization appears to be a separate form of the description of the scalar wave function $A \exp \left(\mathrm{i} k_{0} \psi\right)$, which corresponds to the scalar wave field in the isotropic medium.

Polarization of an electromagnetic wave can be described also by complex polarization angle (CPA) $\gamma=$ $\gamma^{\prime}+\mathrm{i} \gamma^{\prime \prime}$, defined by relation:

$$
\tan \gamma=\frac{\Gamma_{2}}{\Gamma_{1}}
$$

As is shown in [6], the real part of CPA $\gamma^{\prime}$ characterizes orientation of the large semi-axis of the polarization ellipse, whereas the hyperbolic tangent of the imaginary part $\tanh \gamma^{\prime \prime}$ defines ellipticity and CPA obeys a very simple equation

$$
\begin{aligned}
& \frac{\mathrm{d} \gamma}{\mathrm{d} \tau}=\frac{\mathrm{i} k_{0}}{4}\left(\nu_{21}-\nu_{12}\right)+\frac{\mathrm{i} k_{0}}{4}\left[\left(\nu_{22}-\nu_{11}\right) \sin 2 \gamma\right. \\
& \left.-\left(\nu_{12}+\nu_{21}\right) \cos 2 \gamma\right] .
\end{aligned}
$$

A similar equation can be written for complex amplitude ratio (CAR) $\zeta=\frac{\Gamma_{2}}{\Gamma_{1}}[7]$. Within the framework of angular variable technique (AVT) [8], we can also derive equations for angular variables: azimuthal angle of the polarization ellipse $\zeta$ and ellipticity angle $\chi$, whose tangent can be interpreted as a ratio of minor and major axes.

\section{Basic CGO equations}

Like traditional geometrical optics, CGO starts with the presentation of the wave field in the form of Debye expansion in inverse powers of wave number

$$
u(\boldsymbol{r})=\sum_{m=0}^{\infty} \frac{A_{m}(\boldsymbol{r})}{\left(\mathrm{i} k_{0}\right)^{m}} \exp (\mathrm{i} k \psi(\boldsymbol{r})),
$$

where $\psi$ is complex eikonal and $A$ is complex amplitude. Substituting the Debye expansion into the wave equation

$$
\Delta u(\boldsymbol{r})+k_{0}^{2} \varepsilon_{0}(\boldsymbol{r}) u(\boldsymbol{r})=0,
$$

where

$$
\varepsilon_{0}(\boldsymbol{r})=\operatorname{Re}(\varepsilon(\boldsymbol{r}))+\mathrm{i} \operatorname{Im}(\varepsilon(\boldsymbol{r}))=\varepsilon_{0 \mathrm{R}}(\boldsymbol{r})+\mathrm{i} \varepsilon_{0 \mathrm{I}}(\boldsymbol{r}),
$$

and supposing the imaginary part $\varepsilon_{0 \mathrm{II}}(\boldsymbol{r})$ to be small as compared with $\varepsilon_{0 \mathrm{R}}(\boldsymbol{r})$ correspondingly and manipulating $\varepsilon_{0 I}(\boldsymbol{r})$ into quantity of order of $1 / k_{0}$, an eikonal equation is obtained

$$
(\nabla \psi)^{2}=\varepsilon_{0 \mathrm{R}}(\boldsymbol{r})
$$

which depends only on the real part of plasma permittivity and transport equation for amplitude of $m=0$ order in the form

$$
2\left(\nabla A_{0} \nabla \psi\right)+A_{0} \Delta \psi+k_{0} \varepsilon_{0 \mathrm{I}}(\boldsymbol{r}) A_{0}=0,
$$

which contains attenuation factor $k_{0} \varepsilon_{0 \mathrm{I}}(\boldsymbol{r}) A_{0}$.

The CGO method deals with paraxial beams, which implies the smallness of the following two parameters:

$$
\begin{aligned}
& \mu_{\text {DIF }}=\frac{\lambda}{w} \ll 1, \\
& \mu_{\text {PARAX }}=\frac{w}{L} \ll 1,
\end{aligned}
$$

where $w$ is the beam width and $L \sim|\nabla \varepsilon|^{-1}$ is the characteristic scale of the medium inhomogeneity. The diffraction parameter (16) determines the angle of the beam diffraction widening, whereas the "paraxial" parameter (17) requires the beam width to be small as compared with the characteristic scale of the medium inhomogeneity. To generalize CGO method to make it applicable to the case of nonlinear plasma, we introduce two additional small parameters

$$
\begin{aligned}
& \mu_{\mathrm{NL}}=\frac{\lambda}{L_{\mathrm{NL}}} \ll 1, \\
& \mu_{\mathrm{NLPARAX}}=\frac{w}{L_{\mathrm{NL}}} \ll 1,
\end{aligned}
$$

where $L_{\mathrm{NL}} \sim 1 / \varepsilon_{\mathrm{NL}}\left|A_{0}\right|^{2}$ represents the characteristic nonlinear scale, $A_{0}$ denotes the initial amplitude of $\mathrm{GB}$, and parameter $\varepsilon_{\mathrm{NL}}$ denotes nonlinear coefficient of plasma nonlinearity, when we limit ourselves to low signal intensities.

In accordance with paraxial approximation, the permittivity $\varepsilon_{0 \mathrm{R}}=\varepsilon_{0 \mathrm{R}}(\boldsymbol{r})$ on the right-hand side of Eq. (14) can be expanded as a Taylor series in small deviation $\boldsymbol{\xi}=\xi_{1} \boldsymbol{e}_{1}+\xi_{2} \boldsymbol{e}_{2}$ in the form

$$
\begin{aligned}
& \varepsilon_{0 \mathrm{R}}(\boldsymbol{r})=\varepsilon_{0 \mathrm{R}}\left(\boldsymbol{r}_{\mathrm{c}}\right)+\left[\left(\boldsymbol{\xi} \nabla_{\perp}\right) \varepsilon_{0 \mathrm{R}}\right]_{\boldsymbol{r}=\boldsymbol{r}_{\mathrm{c}}} \\
& \quad+\left[\frac{1}{2}\left(\boldsymbol{\xi} \nabla_{\perp}\right)^{2} \varepsilon_{0 \mathrm{R}}\right]_{\boldsymbol{r}=\boldsymbol{r}_{\mathrm{c}}}+\mathrm{O}\left(\mu_{\mathrm{PARAX}}^{3}\right),
\end{aligned}
$$


where $\nabla_{\perp}=\frac{\partial}{\partial \boldsymbol{\xi}}$. We assume that $\boldsymbol{r}_{\mathrm{c}}$ represents the central ray of the wave beam. Following Popov [21], we introduce the curvilinear coordinates $\left(\tau, \xi_{1}, \xi_{2}\right)$ for description of beam evolution by relation [15, 18]:

$$
\boldsymbol{r}=\boldsymbol{r}_{\mathrm{c}}(\tau)+\xi_{1} \boldsymbol{e}_{1}+\xi_{2} \boldsymbol{e}_{2} .
$$

\subsection{Riccati equation for complex curvature}

According to paraxial approximation, pioneered by Luneburg [22], the eikonal $\psi$ can be presented as a sum

$$
\psi\left(\xi_{1}, \xi_{2}, \tau\right)=\psi_{\mathrm{c}}(\tau)+\frac{1}{2} B_{i j}(\tau) \xi_{i} \xi_{j}+\ldots,
$$

where the first term corresponds to the central ray $\psi_{\mathrm{c}}(\tau)=\int \sqrt{\varepsilon_{0}\left(\boldsymbol{r}_{\mathrm{c}}\right)} \mathrm{d} \tau$, and the second term quadratic in $\xi_{i}$ contains the tensor $B_{i j}$ of the wave front curvature. Linear terms $\xi_{i}$ turn out to be zeros in virtue of the Fermat principle, whereas the terms of higher order are small in paraxial approximation [15, 18].

Substituting (22) into the eikonal Eq. (14) leads to Riccati equation for parameters $B_{i j}$, which in fact form the curvature tensor of the wave front $[15,18]$ :

$$
\frac{\mathrm{d} B_{i j}}{\mathrm{~d} \tau}+B_{i k} B_{k j}=\alpha_{i j},
$$

where tensor

$$
\alpha_{i j}(\tau)=\left[\frac{1}{2} \frac{\partial^{2} \varepsilon_{0 \mathrm{R}}(\boldsymbol{r})}{\partial \xi_{i} \partial \xi_{j}}-\frac{3}{4 \varepsilon_{0}(\boldsymbol{r})} \frac{\partial \varepsilon_{0 \mathrm{R}}(\boldsymbol{r})}{\partial \xi_{i}} \frac{\partial \varepsilon_{0 \mathrm{R}}(\boldsymbol{r})}{\partial \xi_{j}}\right]_{\boldsymbol{r}=\boldsymbol{r}_{\mathrm{c}}}
$$

describes here the influence of refraction on complex curvature tensor $B_{i j}$. The real part of matrix $B_{i j}$ characterizes the curvature of the wave front and the principal values $R_{i}$ of the curvature matrix $R_{i j}(\tau)=\operatorname{Re} B_{i j}$ are proportional to the principal curvatures $\kappa_{i}$ :

$$
R_{i}=\kappa_{i} .
$$

At the same time, the principal values $I_{i}$ of matrix $I_{i j}(\tau)=\operatorname{Im} B_{i j}$ determine the principal widths of the Gaussian beam $w_{i}$ in the following way [18]:

$$
I_{i}=\frac{1}{k_{0} w_{i}^{2}} .
$$

\subsection{Transport equation for complex GB amplitude}

In curvilinear coordinates $\left(\tau, \xi_{1}, \xi_{2}\right)$, the transport Eq. (15) for $A=A(\tau)$, takes the form

$$
\begin{aligned}
& \frac{1}{h^{2}} \frac{\mathrm{d} A^{2}}{\mathrm{~d} \tau} \frac{\partial \psi}{\partial \tau}+\left[\frac{1}{h} \frac{\partial}{\partial \tau}\left(\frac{1}{h} \frac{\partial \psi}{\partial \tau}\right)+\frac{\partial^{2} \psi}{\partial \xi_{1}^{2}}+\frac{\partial^{2} \psi}{\partial \xi_{2}^{2}}\right] A^{2} \\
& \quad+k_{0} \varepsilon_{0 \mathrm{I}}(\boldsymbol{r}) A^{2}=0,
\end{aligned}
$$

where

$$
h=\sqrt{\varepsilon(\boldsymbol{r})}\left(1-\frac{\left(\boldsymbol{\xi} \nabla_{\perp}\right) \varepsilon(\boldsymbol{r})}{2 \varepsilon(\boldsymbol{r})}\right)_{\boldsymbol{r}=\boldsymbol{r}_{\mathrm{c}}}
$$

is the Lamé coefficient. In accordance with paraxial approximation and introducing modified amplitude $\tilde{A}=$ $\varepsilon_{\mathrm{c}}^{1 / 4} A$, Eq. (27) takes the following form:

$$
\frac{\mathrm{d} \tilde{A}}{\mathrm{~d} \tau}+\frac{1}{2}\left[\operatorname{Tr} B_{i j}+k_{0} \varepsilon_{0 \mathrm{I}}(\boldsymbol{r})\right] \tilde{A}=0,
$$

where

$$
\operatorname{Tr} B_{i j} \equiv B_{i i}=B_{11}+B_{22}
$$

and $\varepsilon_{\mathrm{c}}$ denotes permittivity measured along propagation trajectory.

By integrating Eq. (29), the complex amplitude of GB takes the following form:

$$
\tilde{A}=\tilde{A}_{0} \exp \left(-\frac{1}{2} \int \operatorname{Tr} B_{i j} \mathrm{~d} \tau\right) \exp \left(-\frac{k_{0}}{2} \int \varepsilon_{0 \mathrm{I}}(\boldsymbol{r}) \mathrm{d} \tau\right) .
$$

Thus, the wave field of the Gaussian beam in a weakly anisotropic medium is presented by the expression

$$
\begin{aligned}
\boldsymbol{E} & =\boldsymbol{\Gamma} \frac{\tilde{A}_{0}}{\sqrt[4]{\varepsilon_{0}\left(\boldsymbol{r}_{\mathrm{c}}\right)}} \exp \left(\mathrm{i} k_{0}\left(\psi_{\mathrm{c}}(\tau)+\frac{1}{2} B_{i j}(\tau) \xi_{i} \xi_{j}\right)\right) \\
& \times \exp \left(-\frac{1}{2} \int \operatorname{Tr} B_{i j} \mathrm{~d} \tau\right) \exp \left(-\frac{k_{0}}{2} \int \varepsilon_{0 \mathrm{I}}(\boldsymbol{r}) \mathrm{d} \tau\right)
\end{aligned}
$$

and taking into account that $B_{i j}(\tau)=R_{i j}(\tau)+\mathrm{i} I_{i j}(\tau)$ we obtain

$$
\begin{aligned}
\boldsymbol{E} & =\boldsymbol{\Gamma} \frac{\tilde{A}}{\sqrt[4]{\varepsilon_{0}\left(\boldsymbol{r}_{\mathrm{c}}\right)}} \exp \left(-\frac{1}{2} k_{0} I_{i j}(\tau) \xi_{i} \xi_{j}\right) \\
& \times \exp \left(\mathrm{i} k_{0}\left(\psi_{\mathrm{c}}(\tau)+\frac{1}{2} R_{i j}(\tau) \xi_{i} \xi_{j}\right)\right) \\
& \times \exp \left(-\frac{1}{2} \int \operatorname{Tr} B_{i j} \mathrm{~d} \tau\right) \exp \left(-\frac{k_{0}}{2} \int \varepsilon_{0 \mathrm{I}}(\boldsymbol{r}) \mathrm{d} \tau\right),
\end{aligned}
$$

where polarization vector $\boldsymbol{\Gamma}$ obeys QIA Eqs. (8), the complex curvature tensor $B_{i j}(\tau)$ satisfies the Riccati Eq. (23) and the amplitude $\tilde{A}$ is determined by Eq. (29) as for paraxial geometrical optics. The essential distinction from real valued paraxial approximation is that Eq. (33) describes diffraction evolution of the Gaussian beam. The examples of diffraction behavior of GB are demonstrated in [17-19], whereas the GB evolution in nonlinear media of the Kerr type is presented in [20].

\section{Gaussian beam diffraction and self-focusing in nonlinear and weakly dissipative magnetized plasma}

The interest in GB evolution in magnetized plasma is driven at least by two problems: microwave plasma diagnostics in large thermonuclear reactors and radio wave propagation in the Earth ionosphere.

\subsection{Evolution of polarization vector and complex polarization angle}

Let us consider an axially symmetric polarized wave beam propagating in cylindrically symmetric nonlinear magnetized and lossy plasma. In such a case, $\boldsymbol{e}_{1}$ and $\boldsymbol{e}_{2}$ are mutually orthogonal unit vectors, orthogonal to the ray $\boldsymbol{r}=\boldsymbol{r}(\tau)$, where coordinate $\tau$ plays a role of the $z$ axis, whereas coordinates $\xi_{1}, \xi_{2}$ along the units vectors $\boldsymbol{e}_{1}, \boldsymbol{e}_{2}$ correspond to $x$ and $y$ axes, respectively.

The anisotropy tensor $\nu_{i j}$ for weakly anisotropic plasma, where $\omega_{\mathrm{p}} / \omega \ll 1$ has the following form [23]: 


$$
\nu_{i j}=\left[\begin{array}{cc}
-u V\left(\sin ^{2} \alpha_{\|} \sin ^{2} \alpha_{\perp}+\cos ^{2} \alpha_{\|}\right) & \mathrm{i} V \sqrt{u} \cos \alpha_{\|}+u V \sin ^{2} \alpha_{\|} \sin \alpha_{\perp} \cos \alpha \\
-\mathrm{i} V \sqrt{u} \cos \alpha_{\|}+u V \sin ^{2} \alpha_{\|} \sin \alpha_{\perp} \cos \alpha_{\perp} & -u V\left(\sin ^{2} \alpha_{\|} \cos ^{2} \alpha_{\perp}+\cos ^{2} \alpha_{\|}\right)
\end{array}\right],
$$

where $u=\left(\omega_{\mathrm{c}} / \omega\right)^{2}=\left(e B_{0}\right)^{2} /(m c \omega)^{2}$ is squared electron cyclotron frequency ratio to the signal frequency, $v=\left(\omega_{\mathrm{p}} / \omega\right)^{2}=4 \pi e^{2} N_{\mathrm{e}} / m \omega^{2}$ is squared plasma frequency ratio to the signal frequency, $N_{\mathrm{e}}$ is the electron density, $e$ and $m$ are the electron charge and mass, parameter $V$ is equal to $V=v /(1-u), B_{0}$ is a static magnetic field, $\alpha_{\|}$stands for the angle between the ray tangent $\boldsymbol{l}$ (in our case there is unit vector along the $z$ axis), and the vector $\boldsymbol{B}_{0}$ and $\alpha_{\perp}$ represent the angle between transverse component $\boldsymbol{B}_{0 \perp}$ and unit vector $\boldsymbol{e}_{1}$ of Popov's coordinate system (Fig. 1).

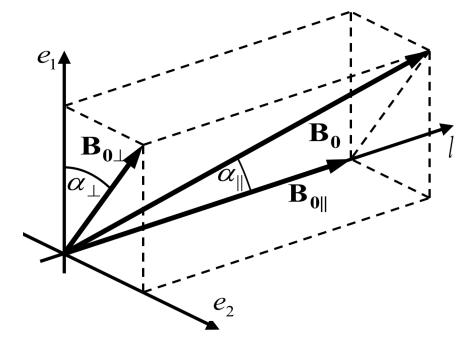

Fig. 1. Orientation of the static magnetic field $\boldsymbol{B}_{0}$ within the framework of the orthogonal coordinate system, formed by the unit vectors $\boldsymbol{e}_{1}, \boldsymbol{e}_{2}$ and by the vector tangent to the ray, which in our case is oriented along the $z$ axis.

As a result, QIA equations for high frequency electromagnetic waves in magnetoactive plasma take a form

$$
\begin{aligned}
& \frac{\mathrm{d} \Gamma_{1}}{\mathrm{~d} z}=\left(\mathrm{i} k_{0} / 2\right) V\left[-u\left(\sin ^{2} \alpha_{\|} \sin ^{2} \alpha_{\perp}+\cos ^{2} \alpha_{\|}\right) \Gamma_{1}\right. \\
& \left.\quad+\left(\mathrm{i} \sqrt{u} \cos \alpha_{\|}+u \sin ^{2} \alpha_{\|} \sin \alpha_{\perp} \cos \alpha_{\perp}\right) \Gamma_{2}\right], \\
& \frac{\mathrm{d} \Gamma_{2}}{\mathrm{~d} z}=\left(\mathrm{i} k_{0} / 2\right) V\left[\left(-\mathrm{i} \sqrt{u} \cos \alpha_{\|}\right.\right. \\
& \left.\quad+u \sin ^{2} \alpha_{\|} \sin \alpha_{\perp} \cos \alpha_{\perp}\right) \Gamma_{1} \\
& \left.\quad-u\left(\sin ^{2} \alpha_{\|} \cos ^{2} \alpha_{\perp}+\cos ^{2} \alpha_{\|}\right) \Gamma_{2}\right] .
\end{aligned}
$$

As it follows from Eq. (34), CPA $\gamma$ obeys the equation

$$
\frac{\mathrm{d} \gamma}{\mathrm{d} z}=\frac{k_{0}}{2} V \sqrt{u} \cos \alpha_{\|}-\frac{\mathrm{i} k_{0}}{4} u V \sin ^{2} \alpha_{\|} \sin \left(2 \gamma-2 \alpha_{\perp}\right) \text {. }
$$

By separating the real and imaginary parts of Eq. (36), the complex valued equation for CPA can be reduced to the system of two real equations for $\gamma_{\mathrm{R}}=\operatorname{Re}(\gamma)$ and $\gamma_{\mathrm{I}}=\operatorname{Im}(\gamma)$ :

$$
\begin{aligned}
& \frac{\mathrm{d} \gamma_{\mathrm{R}}}{\mathrm{d} z}=\frac{k_{0}}{2} V \sqrt{u} \cos \alpha_{\|}-\frac{1}{4} u V \sin ^{2} \alpha_{\|} \cos \left(2 \gamma_{\mathrm{R}}-2 \alpha_{\perp}\right) \\
& \quad \times \sinh 2 \gamma_{\mathrm{I}}, \\
& \frac{\mathrm{d} \gamma_{\mathrm{I}}}{\mathrm{d} z}=\frac{k_{0}}{4} u V \sin ^{2} \alpha_{\|} \sin \left(2 \gamma_{\mathrm{R}}-2 \alpha_{\perp}\right) \cosh 2 \gamma_{\mathrm{I}} .
\end{aligned}
$$

Equations (35)-(38) adequately describe either polarization vector of electromagnetic waves and complex po- larization angle evolution in magnetized plasma and includes all the known phenomena in plasma polarimetry. As a simple and illustrative example, let us describe the Faraday effect and Cotton-Mouton phenomenon using CPA. The Faraday effect linear in magnetic field $\boldsymbol{B}_{0}$ prevails when inequality $\cos \alpha_{\|} \gg \sqrt{u}$ holds, which corresponds to quasi-longitudinal propagation [23]. Under the conditions of quasi-longitudinal propagation, the second term in Eq. (36) is small as compared with the first one and Eq. (36) takes a form

$$
\frac{\mathrm{d} \gamma}{\mathrm{d} z}=\frac{k_{0}}{2} V \sqrt{u} \cos \alpha_{\|},
$$

and has the following solution:

$$
\gamma_{\mathrm{R}}=\gamma_{\mathrm{R}}(0)+\frac{k_{0}}{2} \int_{0}^{z} V \sqrt{u} \cos \alpha_{\|} \mathrm{d} z^{\prime}, \quad \gamma_{\mathrm{I}}=\gamma_{\mathrm{I}}(0) .
$$

In the above equation, $\gamma_{\mathrm{R}}$ describes the Faraday rotation of the polarization angle, taking into account the initial polarization angle $\gamma_{\mathrm{R}}(0)$. The imaginary part $\gamma_{\mathrm{I}}=\gamma_{\mathrm{I}}(0)$ is constant along the symmetry axis, which corresponds to preservation of ellipticity. The Faraday term in Eq. (40) above becomes negligibly small near the orthogonality point $\alpha_{\|}=\pi / 2$, where inequality holds $\cos \alpha_{\|} \ll \sqrt{u}$, corresponding to quasi-transverse propagation. The leading role in Eq. (36) belongs then to the second term, describing the Cotton-Mouton effect, so Eq. (36) becomes

$$
\frac{\mathrm{d} \gamma}{\mathrm{d} z}=-\frac{\mathrm{i} k_{0}}{4} u V \sin ^{2} \alpha_{\|} \sin \left(2 \gamma-2 \alpha_{\perp}\right),
$$

and has the solution

$$
\begin{aligned}
& \gamma_{\mathrm{R}}=\gamma_{\mathrm{R}}(0), \\
& \gamma_{\mathrm{I}}=-\frac{k_{0}}{4} \int_{0}^{\tau} u V \sin ^{2} \alpha_{\|} \sin \left(2 \gamma-2 \alpha_{\perp}\right) \mathrm{d} z,
\end{aligned}
$$

where the imaginary part is responsible for the transformation of a linearly polarized wave into an elliptically polarized wave.

\subsection{Evolution of the $G B$ width, wave front curvature and complex amplitude}

For an axially symmetric wave beam in cylindrically symmetric plasma, CGO suggests the following form of solution:

$$
\begin{aligned}
& E(\rho, z)=A \exp (\mathrm{i} k \psi) \\
& \quad=A(z) \exp \left(\mathrm{i} k\left(z+B(z) \rho^{2} / 2\right)\right),
\end{aligned}
$$

where $\psi$ is complex-valued eikonal, $\rho=\sqrt{x^{2}+y^{2}}$ is a distance from the $z$ axis and complex parameter $B=$ $R+\mathrm{i} I$ has now the form

$$
R=\kappa(z), \quad I=\frac{1}{k_{0} w^{2}(z)},
$$

where, as in Sect. 3, $\kappa=\kappa(z)$ is the wave front curvature and $w=w(z)$ denotes the GB width. Substituting (44) 
in Eq. (43), we obtain the Gaussian beam of the form

$$
\begin{aligned}
& E(\rho, z)=A(z) \exp \left(-\frac{\rho^{2}}{2 w^{2}(z)}\right) \exp \left(-\int \kappa \mathrm{d} z\right) \\
& \quad \times \exp \left(\mathrm{i} k\left(z+\kappa(z) \frac{\rho^{2}}{2}\right)\right) \exp \left(-\mathrm{i} \int \mathrm{d} z / k_{0} w^{2}\right) .
\end{aligned}
$$

Let us consider the GB (45) propagating in nonlinear dissipative plasma whose isotropic part of dielectric permittivity (1) has the form [24-28]:

$$
\varepsilon_{0}(z, \rho)=\varepsilon_{0 \mathrm{~L}}+\frac{\omega_{\mathrm{p}}^{2}}{\omega^{2}}\left[1-\exp \left(-\varepsilon_{\mathrm{NL}}|E|^{2}\right)\right]+\mathrm{i} \varepsilon_{0 \mathrm{I}},
$$

where

$$
\varepsilon_{0 \mathrm{~L}}=1-\frac{\omega_{\mathrm{p}}^{2}}{\omega^{2}}
$$

is the linear part of plasma permittivity, parameter $\varepsilon_{\mathrm{NL}}$ denotes nonlinear coefficient of ponderomotive plasma nonlinearity and we assume that the imaginary part of plasma permittivity is constant $\varepsilon_{0 \mathrm{I}}=$ const. A more general case when $\varepsilon_{0 \mathrm{I}}$ is a function of $E E^{*}$ is very difficult to pursue mathematically, hence we limit ourselves to the case when $\varepsilon_{0 \mathrm{I}}$ is field independent (i.e. absorption is linear). The case of nonlinear plasma absorption was analyzed in [29]. Further modifications of Eq. (23) and Eq. (29) for the case of linear absorption, where $\varepsilon_{0 I}=$ const leads to the evolution of flux energy in nonlinear dissipative plasma in the form

$$
w^{2}|A|^{2}=w_{0}^{2}\left|A_{0}\right|^{2} \exp \left(-k_{0} \varepsilon_{0 \mathrm{I}} z\right),
$$

where $w_{0}$ is the initial beam width and $A_{0}$ denotes the initial amplitude. Taking advantage of the CGO procedure presented in Sect. 3, the eikonal equation can be presented in coordinates $(\rho, z)$, and after expansion of permittivity $\varepsilon_{0}(z, \rho)$ in the Taylor series in $\rho$ in the vicinity of symmetry axis $z$, we obtain a Riccati equation

$$
\frac{\mathrm{d} B}{\mathrm{~d} z}+B^{2}=\alpha \text {. }
$$

Parameter $\alpha$ for axially symmetric medium with permittivity (46), taking into account (44) and (48) is equal to

$$
\alpha(z)=-\frac{\omega_{\mathrm{p}}^{2} p}{\omega^{2} w^{4}(z)} \exp \left(-\frac{p}{w^{2}(z)}\right) \exp \left(-k_{0} \varepsilon_{0 \mathrm{I}} z\right),
$$

where $p=\gamma w_{0}^{2}\left|A_{0}\right|^{2}$. The Riccati Eq. (49) is equivalent to the set of two equations for the real and imaginary parts of the complex curvature $B=R+\mathrm{i} I$ :

$$
\left\{\begin{array}{l}
\frac{\mathrm{d} R}{\mathrm{~d} z}+R^{2}-I^{2}=\alpha, \\
\frac{\mathrm{d} I}{\mathrm{~d} z}+2 R I=0 .
\end{array}\right.
$$

Substituting $R=\kappa, I=1 / k_{0} w^{2}$ in the second of Eqs. (51) we obtain the known relation between the beam width $w$ and the wave front curvature $\kappa$, derived in [20]:

$$
\kappa=\frac{1}{w} \frac{\mathrm{d} w}{\mathrm{~d} z} .
$$

Substituting the next Eq. (52) into the first of Eqs. (51) we obtain the ordinary differential equation of the second order for $\mathrm{GB}$ width

$$
\begin{aligned}
& \frac{\mathrm{d}^{2} w}{\mathrm{~d} z^{2}}+\frac{\omega_{\mathrm{p}}^{2} p}{\omega^{2} w^{3}(z)} \exp \left(-\frac{p}{w^{2}(z)}\right) \exp \left(-k_{0} \varepsilon_{0 \mathrm{I}} z\right) \\
& \quad=1 /\left(k_{0}^{2} w^{3}\right) .
\end{aligned}
$$

For the following parameters: $\omega_{\mathrm{p}}^{2} / \omega^{2}=0.2, w_{0}=$ $0.1 \mathrm{~mm}, \lambda=0.01 \mathrm{~mm}$ and $\varepsilon_{0 \mathrm{I}}=10^{-4}$ Eqs. $(52,53)$ together with Eq. (48) are solved numerically. The solutions are shown in Figs. 2-7.

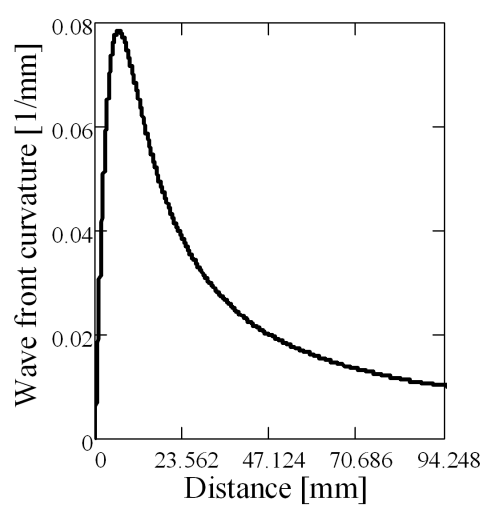

Fig. 2. Wave front curvature evolution in under-critical regime of nonlinear cylindrical plasma, where $\varepsilon_{\mathrm{NL}}\left|A_{0}\right|^{2}=10^{-4}\left(P=0.4 P_{\text {crit }}\right), \mu_{\mathrm{A}}=0.2, \mu_{\mathrm{GO}}=$ $0.02, \mu_{\mathrm{DIF}}=0.1, \mu_{\mathrm{PARAX}}=0.2, \mu_{\mathrm{NL}}=1 / 30$, and $\mu_{\text {NLPARAX }}=0.01$. The plot is shown in the range from 0 to one diffraction distance $L_{\mathrm{D}}=k_{0} w_{0}^{2}$.

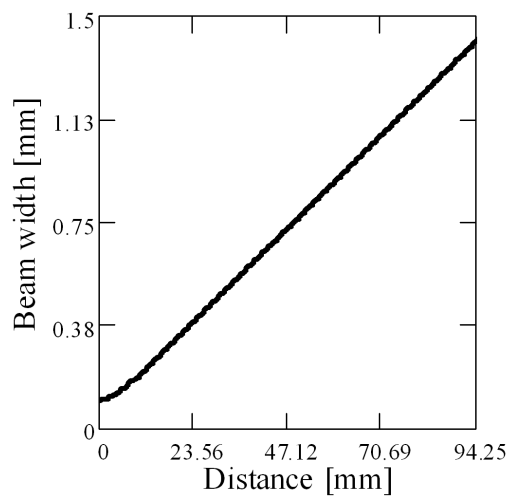

Fig. 3. Beam width evolution in under-critical regime of nonlinear cylindrical plasma, where $\varepsilon_{\mathrm{NL}}\left|A_{0}\right|^{2}=$ $10^{-4}\left(P=0.4 P_{\text {crit }}\right), \mu_{\mathrm{A}}=0.2, \mu_{\mathrm{GO}}=0.02, \mu_{\mathrm{DIF}}=0.1$ $\mu_{\mathrm{PARAX}}=0.2, \mu_{\mathrm{NL}}=1 / 30$, and $\mu_{\mathrm{NLPARAX}}=0.01$. The plot is shown in the range from 0 to 15 diffraction distances.

It is notable in Figs. 2-4 that in under-critical regime, where $P<P_{\text {crit }}$, the wave front curvature initially increases to maximal value and next decreases asymptotically. For such a case, the GB width increases unlimitedly, whereas the absolute value of complex amplitude decreases asymptotically to zero. In over-critical regime Figs. 5-7, where $P>P_{\text {crit }}$, the wave front curvature oscillates with decreasing amplitude of these oscillations. The GB width oscillates with increasing amplitude of these oscillations. The modulus of complex amplitude initially decreases to the minimal value and next increases to the maximal value, after that decreasing asymptotically to zero again. 


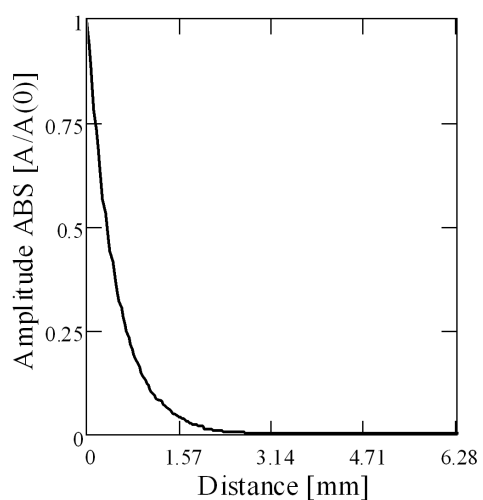

Fig. 4. Absolute value $|A| /\left|A_{0}\right|$ evolution in under-critical regime of nonlinear cylindrical plasma, where $\varepsilon_{\mathrm{NL}}\left|A_{0}\right|^{2}=10^{-4}\left(P=0.4 P_{\text {crit }}\right), \mu_{\mathrm{A}}=0.2, \mu_{\mathrm{GO}}=$ $0.02, \mu_{\mathrm{DIF}}=0.1, \mu_{\mathrm{PARAX}}=0.2, \mu_{\mathrm{NL}}=1 / 30$, and $\mu_{\text {NLPARAX }}=0.01$. The plot is shown in the range from 0 to one diffraction distance.

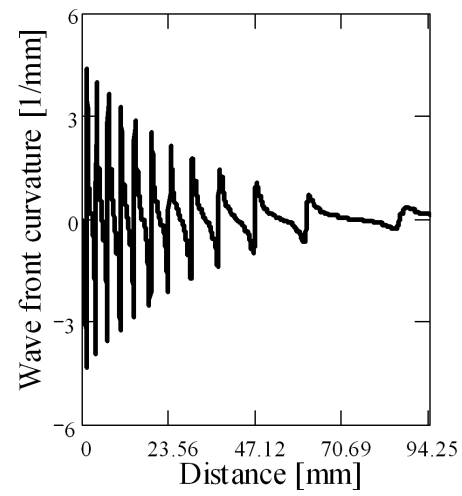

Fig. 5. Wave front curvature evolution in overcritical regime of nonlinear cylindrical plasma, where $\gamma\left|A_{0}\right|^{2}=$ $10^{-3}\left(P=4 P_{\text {crit }}\right), \mu_{\mathrm{A}}=0.2, \mu_{\mathrm{GO}}=0.02, \mu_{\mathrm{DIF}}=$ $0.1, \mu_{\mathrm{PARAX}}=0.2, \mu_{\mathrm{NL}}=0.1$, and $\mu_{\mathrm{NLPARAX}}=1 / 30$. The plot is shown in the range from 0 to 15 diffraction distances.

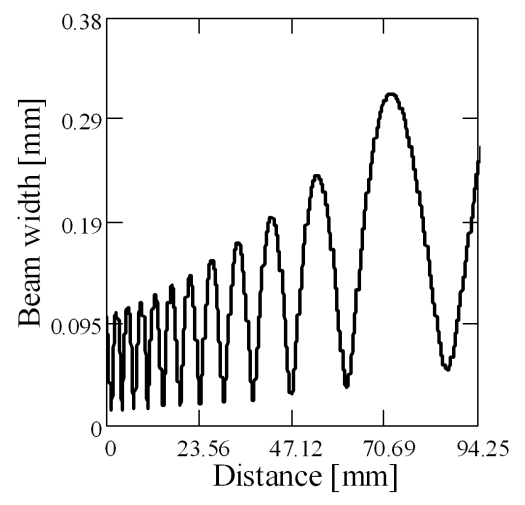

Fig. 6. Beam width evolution in overcritical regime of nonlinear cylindrical plasma, where $\gamma\left|A_{0}\right|^{2}=10^{-3}(P=$ $\left.4 P_{\text {crit }}\right), \mu_{\mathrm{A}}=0.2, \mu_{\mathrm{GO}}=0.02, \mu_{\mathrm{DIF}}=0.1, \mu_{\mathrm{PARAX}}=$ $0.2, \mu_{\mathrm{NL}}=0.1$, and $\mu_{\mathrm{NLPARAX}}=1 / 30$. The plot is shown in the range from 0 to 15 diffraction distances.

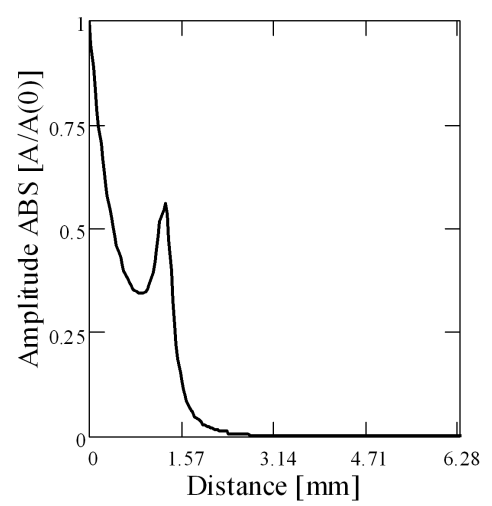

Fig. 7. Absolute value $|A| /\left|A_{0}\right|$ evolution in overcritical regime of nonlinear cylindrical plasma, where $\gamma\left|A_{0}\right|^{2}=10^{-3}\left(P=4 P_{\text {crit }}\right), \mu_{\mathrm{A}}=0.2, \mu_{\mathrm{GO}}=0.02$, $\mu_{\mathrm{DIF}}=0.1, \mu_{\mathrm{PARAX}}=0.2, \mu_{\mathrm{NL}}=0.1$, and $\mu_{\mathrm{NLPARAX}}=$ $1 / 30$. The plot is shown in the range from 0 to one diffraction distance.

\section{Conclusions}

The paper presents a combination of CGO and QIA of geometrical optics method, which allows to describe both polarization and diffraction changes of the wave field. CGO describes evolution of the GB width and wave front curvature under the influence of diffraction on the basis of the Riccati type ordinary differential equation of the first order, whereas complex amplitude of the Gaussian beam can be found from the transport equation which is also a first order ordinary differential equation. At the same time, QIA determines polarization of the electromagnetic wave on the basis of the first order coupled equations for the components of polarization vector, or on the basis of the first order differential equation for complex polarization angle. As a result, the combined QIA/CGO approach reduces diffraction and polarization evolution of the Gaussian beam in the nonlinear plasma to solution of the ordinary differential equations of the first order. These simple and effective approaches are illustrated by the example of Gaussian beam propagation and diffraction in magnetized cylindrical plasma with ponderomotive nonlinearity and absorption. The presented method also finds application in ionosphere plasma, or any other weakly anisotropic nonlinear media.

\section{Acknowledgments}

This work was supported by Association IPPLM-EURATOM, project P-12. The authors thank an anonymous reviewer for detailed analyses of the paper and valuable comments.

\section{References}

[1] Yu.A. Kravtsov, N.Y. Zhu, Theory of Diffraction: Heuristic Approaches, Alpha Science International, 2010.

[2] A.A. Fuki, Yu.A. Kravtsov, O.N. Naida, Geometrical Optics of Weakly Anisotropic Media, Gordon \& Breach, London 1997.

[3] Yu.A. Kravtsov, Yu.I. Orlov, Geometrical Optics of Inhomogeneous Media, Springer Verlag, Berlin 1990. 
[4] Yu.A. Kravtsov, Geometrical Optics in Engineering Physics, Alpha Science, Harrow 2005.

[5] Yu.A. Kravtsov, B. Bieg, K.Yu. Bliokh, J. Opt. Soc. Am. A 24, 3388 (2007).

[6] Z.H. Czyz, B. Bieg, Yu.A. Kravtsov, Phys. Lett. A 368, 101 (2007).

[7] Yu.A. Kravtsov, B. Bieg, J. Plasma Phys. 76, 795 (2010).

[8] A. Yu, B. Kravtsov, B. Bieg, Central European J. Phys. 8, 273 (2010).

[9] Yu.A. Kravtsov, J. Chrzanowski, D. Mazon, Europ. Phys. J. D 63, 135 (2011).

[10] Yu.A. Kravtsov, Radiophys. Quant. Electron. 10, 719 (1967).

[11] J.B. Keller, W. Streifer, J. Opt. Soc. Am. 61, 40 (1971).

[12] G.A. Deschamps, Electron. Lett. 7, 684 (1971).

[13] Yu.A. Kravtsov, G.W. Forbes, A.A. Asatryan, in: Progress in Optics, Ed. E. Wolf, Vol 39, Elsevier, Amsterdam 1999, p. 3.

[14] S.J. Chapman, J.M. Lawry, J.R. Ockendon, R.H. Tew, SIAM Rev. 41, 417 (1999).

[15] Yu.A. Kravtsov, P. Berczynski, Stud. Geophys. Geod. 51, 1 (2007).

[16] Yu.A. Kravtsov, P. Berczynski, Wave Motion 40, 23 (2004).

[17] P. Berczynski, Yu.A. Kravtsov, Phys. Lett. A 331, 265 (2004).
[18] P. Berczynski, K.Yu. Bliokh, Yu.A. Kravtsov, A. Stateczny, J. Opt. Soc. Am. A 23, 1442 (2006).

[19] P. Berczynski, Yu.A. Kravtsov, G. Zeglinski, Cent. Eur. J. Phys. 6, 603 (2008).

[20] P. Berczynski, Yu.A. Kravtsov, A.P. Sukhorukov, Physica D: Nonlin. Phenom. 239, 241 (2010).

[21] M.M. Popov, Vestnik Leningradskogo Universiteta (Bull. Leningrad Univ.) 22, 44 (1969).

[22] R.K. Luneburg, Mathematical Theory of Optics, University of California Press, Berkeley 1964.

[23] V.I. Ginzburg, Propagation of Electromagnetic Waves in Plasma, Gordon and Breach, New York 1970.

[24] C.E. Max, The Physics of Fluids 19, 74 (1976).

[25] J.F. Lam, B. Lipman, F. Tappert, Phys. Fluids 20, 1176 (1977).

[26] D. Anderson, Phys. Scr. 18, 35 (1978).

[27] D.P. Tewari, R.R. Sharma, J. Phys. D, Appl. Phys. 12, 1019 (1979).

[28] T.S. Gill, R. Mahajanand, R. Kaur, Phys. Plasmas 18, 033110 (2011).

[29] M.S. Sodha, D.P. Tewari, A. Kumar, V.K. Tripathi, J. Phys. D, Appl. Phys. 7, 345 (1974). 